\title{
Content Determination for Natural Language Descriptions of Predictive Bayesian Networks
}

\author{
M. Pereira-Fariña ${ }^{a}$ and Alberto Bugarín ${ }^{b}$ \\ ${ }^{a}$ Departamento de Filosofía e Antropoloxía Social, \\ Universidade de Santiago de Compostela, martin.pereira@usc.es \\ ${ }^{b}$ Centro Singular de Investigación en Tecnoloxías Intelixentes (CiTIUS), \\ Universidade de Santiago de Compostela, alberto.bugarin.diz@usc.es
}

\begin{abstract}
The dramatic success of Artificial Intelligence and its applications has been accompanied by an increasing complexity, which makes its comprehension for final users more difficult and damages their trustworthiness. Within this context, the emergence of Explainable AI aims to make intelligent systems decisions more transparent and understandable for human users. In this paper, we propose a framework for the explanation of predictive inference in Bayesian Networks (BN) in natural language to non-specialized users. The model represents the embedded information in the BN by means of (fuzzy) quantified statements and reasons using the a fuzzy syllogism. The framework provides how this can be used for the content determination stage in Natural Language Generation explanation systems for BNs. Through a number of realistic scenarios of use examples, we show how the generated explanations allows the user to trace the inference steps in the approximate reasoning process in predictive BNs.
\end{abstract}

Keywords: Content Determination in natural language generation, Linguistic descriptions, Fuzzy syllogism.

\section{Introduction}

The dramatic success of applications in Artificial Intelligence (AI) has highlighted the importance of making the decisions and actions of intelligent systems more understandable for their users. This is precisely the main goal of the Explainable AI (XAI) paradigm, which has been catalysed by workshops and conferences $[2,1]$. The two main goals of XAI are [3]:

- To develop more transparent models without affecting their effectiveness and accuracy;
- To deliver understandable and trustworthy explanations.

Furthermore, the European Union's General Data Protection Regulation [11] actually has created what many authors consider a "de-facto right to explanation". From April 2018, whereby a user can ask for an explanation of an algorithmic decision that was made about him/her. This legislation is starting to fuel the explanation perspective in AIbased systems and applications, which also will contribute to clarify the open debate about possible biases in these systems within the general framework of Fairness, Accountability, Transparency and Ethics (FATE) perspective in AI in general.

In this paper, we focus on automatic linguistic explanation in Bayesian Networks [7] (BN), since understanding of the underlying approximate knowledge representation and reasoning mechanisms (e.g. Conditional Probability Tables, belief updating, message passing algorithms, etc.) is not straightforward for inexperienced users. Upon BNs model, a number of software applications have been proposed for (mostly in a graphic way) helping users and designers to build BN-based models for different application areas. For instance, Promedas [17] is a probabilistic decision support system for medical diagnosis with two goals: i) to offer patients specific diagnosis advice and, ii) educational purposes. In both cases, for a regular patient is not enough to see the knowledge coded in a graph but he/she also needs to understand the reasons that support the output of the system in order to be responsive. On the other hand, several fallacies and misunderstandings that inexperienced users commit when using or applying BNs $[6,5]$ are described in the literature; therefore, explainability is still being an open question in this type of formalisms.

Other software tools for BNs [9] help to visualise the knowledge represented within a $\mathrm{BN}$ and to perform the calculations associated with the approximate reasoning process (belief propagation and updating), but they do not provide meaningful information about the logic underlying the model. In other words, graphical tools support the elaboration of the BNs but they do not address interpretability. 
The challenge is to design a computational system that will be able to generate an explanatory narrative of the reasoning process in a register of language which is adequate for non-experienced users.

The focus of this paper is on one of the key stages of Natural Language Generation (NLG) systems, which is Content Determination [14]. Content determination in this context consists in extracting the most relevant information to be included in the final explanations in natural language which are conveyed to the users. The content determined in this stage is usually represented in terms of an intermediate language which is subsequently realised in plain natural language in other stages (planning, micro-planning and linguistic realisation). Template-based NLG approaches [16] usually merge these three subsequent stages into a single one (linguistic realisation), since templates take care of both planning and micro-planning simultaneously in a straightforward manner. Our content determination approach is based on the fuzzy syllogistic theory [13], which allows us to build a Knowledge Base (KB), made up of binary Quantified Statements (QS), that comprises the relevant information which supports the $\mathrm{BN}$ reasoning. Using the BN topology, we determine the key contents for a template-based short narrative based on the semantic concept of "causal chaining" in different levels of depth, where the user can navigate from the query variable to the root nodes in order to know how each step is involved in the belief updating process. The obtained QSs in the content determination stage can be integrated in a template-based NLG system with the linguistic realisation stage, in order to obtain an automatic system for the generation of explanations of BN. However, this task is out of the aim of this paper and will be addressed as future work.

In this paper, we describe our framework for the case of predictive reasoning in BNs, both without fixed evidences and with them. For the sake of simplicity, we illustrate the behaviour of this model by means of a well-known example in the $\mathrm{BN}$ literature [8].

The paper is organised as follows: Section 2 describes the main features of the syllogistic theory that supports how can probabilities can be worded as binary quantified statements; Section 3 describes our concept of explainability in BNs and how the text with the explanation can be generated, both in predictive inference without evidence and with it; and, finally, Section 4, summarises our main conclusions and propose some tasks for future work.

\section{Syllogistic Expression of Conditional Probabilities}

Probability and uncertainty are not just mathematical concepts, but they are very common in natural language. Thus, qualitative expressions such as very high probability or unlikely or quantitative ones such as $100 \%$ or between $30 \%$ and $35 \%$ are frequently used in everyday language and reasoning for dealing with situations involving uncertainty.

This idea applies to BNs as well. Schwartz [15] and Oaksford and Charter [10] defined the equivalence between the mathematical notation of probability $(P(X)=Z)$, conditional probability $(P(X \mid Y)=Z)$ and binary QS. QSs are of the form $Q S T$ are $P T$, where $Q$ stands for a probability $Z$, which can be quantitative (such as the crisp " $5 \%$ ", the fuzzy "around $20 \%$ ", etc.) or qualitative ("a few", "most", etc.); $S T$ is the the subject-term of the statement (a single term or a Boolean combination) and stands for the universe of reference in the case of a priori probabilities $(P(X)=Z)$ or $Y$ in the case of conditional probabilities $(P(X \mid Y)=Z)$; and, $P T$ is the predicate-term (which can also be a single one or a Boolean combination) and stands for $X$. For instance, the a priori probability $P($ Cancer $)=1.16 \%$ can be expressed as " $1.16 \%$ of people have cancer" whilst the Conditional Probability $P($ Cancer $\mid$ Smoker $)=5 \%$ can be expressed as "5\% of smokers have cancer" or "5\% of people who are smokers have cancer".

Quantified statements employ a set-based semantics instead of a Bayesian one. Therefore, each statement is denoting a particular set or subset of the universe and the quantifier heading the sentence its cardinality. From the point of view of human understanding of reasoning, its interpretation is not a trivial step but it affects the persuasiveness of the argument for the user [4]; i.e., how the information is presented has cognitive effects on the addressee.

Based on these assumptions, Pereira-Fariña et al. [13] enhanced the theory of syllogistic logic to handle arguments with more than two fuzzy premises and three terms, which opens the possibility of using fuzzy syllogistics for developing a linguistic version of BNs [12]. The general inference pattern for the generalised fuzzy syllogism is described as follows:

$$
\begin{array}{cl}
P R 1: & Q_{1} L_{1,1} \text { are } L_{1,2} \\
P R 2: & Q_{2} L_{2,1} \text { are } L_{2,2} \\
\ldots & \\
P R N: & Q_{N} L_{N, 1} \text { are } L_{N, 2} \\
\hline C: & Q_{C} L_{C, 1} \text { are } L_{C, 2}
\end{array}
$$

where $P R n, n=1, \ldots, N$ denote the premises and $C$ the conclusion. In any premise, $P R n, Q_{n}$ denotes are its (fuzzy) quantifier, $L_{n, j}, j=1,2$ denote an arbitrary Boolean combination among the terms considered in the syllogism (subject-term $S T$ for $j=1$, predicate-term $P T$ for $j=2$ ). Similarly, for the Conclusion $C, Q_{C}$ stands for its quantifier (which is the value to be calculated) and $L_{C, 1}$ and $L_{C, 2}$ stand respectively for its subject-term and predicate-term.

This schema has the same structure of any propositional logic argument, where the premises convey the available 
information and, the conclusion, the variable of interest (query). A critical step for defining the equivalent syllogism to a $\mathrm{BN}$ is how the information conveyed in a $\mathrm{BN}$ has to be expressed. To the best of our knowledge, this task has not been addressed yet in the literature.

In Table 1 we define the five steps procedure to be followed for converting a $\mathrm{BN}$ into an equivalent $\mathrm{KB}$ made up of binary QSs. Once the universe of reference $U$ is set (step 1), we distinguish two well-differentiated parts in a $\mathrm{BN}$ that must be independently analysed: root nodes (steps 2 and 3 ) and non-root nodes (step 4).

Root-nodes do not have parents and, consequently, the corresponding variables are not conditioned by others. The values of these variables are associated with a priori probabilities. As described in Table 1-step 2, the ST of the QSs is $U$ and their $P T$ are the pairs variable-value. The values of the crisp quantifiers are directly extracted from the $a$ priori probabilities. The independence relationship between the variables of the root-nodes is implicit in the $\mathrm{BN}$, but it has to be made explicit in order to obtain a full representation of it, and a consistent explanation for the reasoning process. This is addressed in Table 1-step 3. The quantifier is calculated here using the product rule because of the independence condition.

Table 1-step 4 deals with non-root nodes. Here, the $S T$ of the $Q S$ are the variables in the parent nodes of the $P T$. The values of the quantifiers are now taken from the Conditional Probability Tables (CPT) in the BN.

The last point of the procedure addresses the wording of the QS in the KB; i.e., the intermediate language of the content determination step. Each one of the QS follows the form $Q$ $S T$ are $P T$, where $Q$ stands for the corresponding quantifier and $S T$ and $P T$ stand for Subject-term and Predicate-term correspondingly.

For illustrative purposes, we consider in Fig. 1 a handbook example of BN about the medical diagnosis of cancer [8, p. 29-36]. Table 2 contains the equivalent KB made up of binary QSs obtained as a result of applying the procedure described in Table 1. We assume the fuzzy protoform " $Q X$ is $A$ and $Y$ is $B$ and..." which is represented in Table 2) with the following intermediate language ntuple notation: $(Q, X$ is $A, Y$ is $B, \ldots)$. Three different blocks or types of QSs are generated, which correspond to: i) variables related to root-nodes pollution (QS1-QS2) and smoker (QS3-QS4), ii) independence condition between these variables (QS5-QS8) and iii) non-root nodes variables cancer (QS9-QS16), XRay (QS17-QS20) and dyspnoea (QS21-QS24). The KB in Table 2 describes in an intermediate language all the relevant information that is explicitly or implicitly included in the $\mathrm{BN}$ of the example of Fig. 1. Chaining among QSs provide explanation paths for the approximate reasoning in the $\mathrm{BN}$, as we show in the

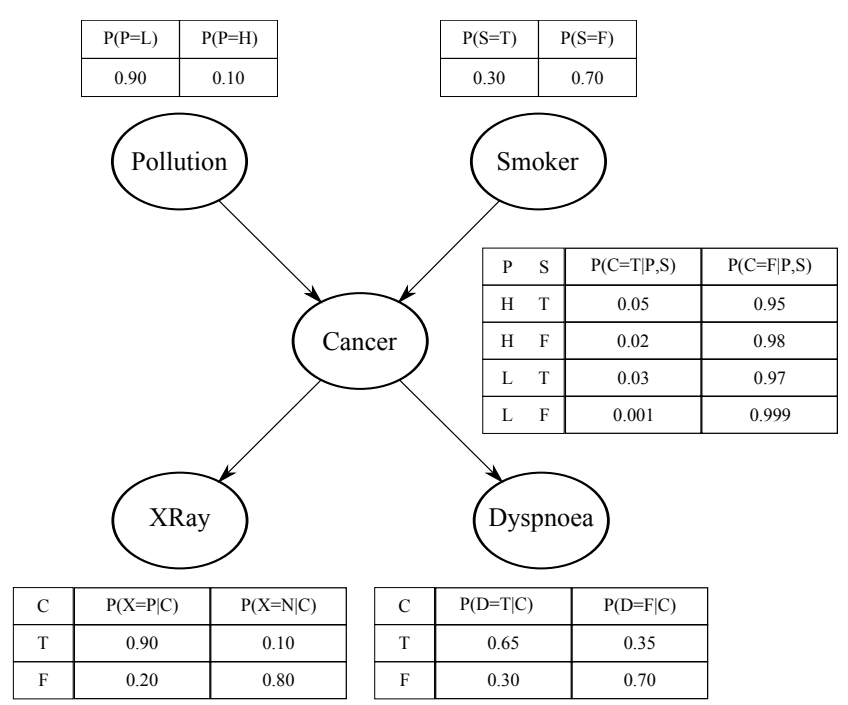

Figure 1: A BN for the lung cancer problem described in [8].

example scenarios in Section 3.2. ${ }^{1}$

\section{Explainability of Bayesian Predictive Inference}

Forward or predictive reasoning can be executed with or without evidence. Without evidence, it is the immediate belief propagation through the network once the values of the CPTs are defined, and, with evidence, a particular variable in one of the nodes takes a concrete value and this triggers a new belief updating process [8]. From the point of view XAI and a final user, inference with evidence is most interesting because it allows the user to define specific conditions and see the updated probability explicable to them.

In this section, we will describe the general procedure for the generation of the linguistic explanation and then illustrate it using the BN in Fig. 1. This network is composed of five nodes, with two root nodes, all containing bi-valued variables (Pollution $=\{$ Low, High $\}$, Smoker $=\{$ True, False $\}$, Cancer $=\{$ True, False $\}$, $X$ Ray $=\{$ Positive, Negative $\}$ and Dyspnoea $=$ $\{$ True, False $\}$ ).

\subsection{General Procedure}

Predictive inference in BNs can involve evidence or not. In the former case, and without loss of generality, let us consider a node $N$ in a BN, associated to variable $X$ and let $x$ be one of its values. The procedure, in this case, will obtain the QSs associated with the BN which explain the inferred

${ }^{1}$ Furthermore, performing syllogistic reasoning in the $\mathrm{KB}$ in Table 2, is equivalent to predictive reasoning in BNs. For instance, one of the QS obtained as a conclusion is " $1.163 \%$ of people have cancer." 
Let $U$ be the universe of reference, $X_{i}$ the $i$-th probabilistic variable (associated to the corresponding node of the $\mathrm{BN}), V A L_{X_{i}}=\left\{x_{i, n_{i}} ; n_{i}=1, \ldots, N_{i}\right\}$ the set of its linguistic values. Each linguistic value is denoted $x_{i, n_{i}}$ and the number of these linguistic values is $N_{i}$.

1. BUILDING THE STATEMENTS CORRESPONDING TO ROOT NODES (A PRIORI PROBABILITIES).

For each variable $X_{i}$ associated to a root node and $\forall x_{i, n_{i}} \in V A L_{X_{i}}$, a binary quantified statement is added to the $\mathrm{KB}$, with

- Quantifier $=P\left(X_{i}=x_{i, n_{i}}\right)$

- Subject-term = noun corresponding to the $U$

- Predicate-term = noun phrase corresponding to the variable and the value that it takes; i.e., " $X_{i}$ is $x_{i, n_{i}}$ "

Therefore, the total number of statements to be generated in this step is $\sum_{i=1}^{I_{R N}} N_{i}$, being $I_{R N}$ the number of root nodes.

2. BUILDING THE STATEMENTS CORRESPONDING TO ROOT NODES (INDEPENDENCE CONDITIONS). For each different combination of all the values of the $I$ variables $X_{i}$ associated to root nodes, a binary quantified statement is added to the $\mathrm{KB}$, with

- Quantifier $=\prod_{i=1}^{I} P\left(X_{i}=x_{i, n_{i}}\right)$

- Subject-term = noun corresponding to the $U$

- Predicate-term $=$ conjunction using and with all the corresponding values; i.e., " $X_{1}$ is $x_{1, n_{1}}$ and $\ldots$ and $X_{I}$ is $x_{I, n_{I}}$ "

Therefore, the total number of statements to be added in this step is $\prod_{i=1}^{I_{R N}} N_{i}$, being $I_{R N}$ the number of root nodes.

3. BUILDING THE STATEMENTS CORRESPONDING TO NON-ROOT NODES.

Let $X_{i}$ be a variable associated to an intermediate node and $P_{X_{i}}=\left\{X_{1}, \ldots, X_{K}\right\}$ the set of variables associated to its $K$ parents. The corresponding values for each $X_{k}, k=1, \ldots, K$ is denoted as $V A L_{X_{k}}=$ $\left\{x_{k, n_{k}} ; n_{k}=1, \ldots, N_{k}\right\}$. For all $n_{i} \in\left\{1, \ldots, N_{i}\right\}$, for each different combination of the values of the variables $X_{k}$ associated to the parent nodes, a binary quantified statement is added to the KB, with

- Quantifier $=P\left(X_{i}=x_{i, n_{i}} \mid X_{1}=x_{1, n_{1}}, \ldots, X_{K}=x_{K, n_{K}}\right)$

- Subject-term $=$ the variables corresponding to the parent nodes: i.e., " $X_{1}$ is $x_{1, n_{1}}$ and $\ldots$ and $X_{K}$ is $x_{K, n_{K}}$ "

- Predicate-term = the variables corresponding to the child node; i.e., " $X_{i}$ is $x_{i, n i}$ "

These QS make explicit the independence conditions between variables associated to root nodes. Therefore, the total number of statements to be added in this step is $\sum_{k=1}^{K} N_{k}$, being $K$ the number of parent nodes.

4. WORDING OF THE QS IN THE KB.

Each of the binary quantified statements added in the previous steps are worded in the usual form:

$$
Q S T \text { are } P T,
$$

where $Q, S T$ and $P T$ are, respectively, the corresponding previously defined Quantifier, Subject-term and Predicate-term

Table 1: Procedure for the Description of a Bayesian Network in terms of Binary Quantified Statements (Content determination stage). 


\begin{tabular}{|c|c|c|}
\hline Procedure of Table 1 & Statement & Content determination $(Q X$ is $A$ and $Y$ is $B$ and... $)$ \\
\hline \multirow{4}{*}{ Step 1: Statements for root-nodes } & QS1 & $(90 \%$, people, Pollution is Low $)$ \\
\hline & QS2 & $(10 \%$, people, Pollution is High $)$ \\
\hline & QS3 & $(30 \%$, people, Smoker is True) \\
\hline & QS4 & $(70 \%$, people, Smoker is False) \\
\hline \multirow{4}{*}{$\begin{array}{l}\text { Step 2: Statements for } \\
\text { independence of root-nodes }\end{array}$} & QS5 & (27\%, people, Pollution is Low, Smoker is True) \\
\hline & QS6 & $(63 \%$, people, Pollution is Low, Smoker is False) \\
\hline & QS7 & (3\%, people, Pollution is High, Smoker is True) \\
\hline & QS8 & $(7 \%$, people, Pollution is High, Smoker is False) \\
\hline \multirow{16}{*}{$\begin{array}{l}\text { Step 3: Statements corresponding } \\
\text { to non-root nodes }\end{array}$} & QS9 & (5\%, people, Pollution is High, Smoker is True, Cancer is True) \\
\hline & QS10 & (95\%, people, Pollution is High, Smoker is True, Cancer is False) \\
\hline & QS11 & $(2 \%$, people, Pollution is High, Smoker is False, Cancer is True) \\
\hline & QS12 & (98\%, people, Pollution is High, Smoker is False, Cancer is False) \\
\hline & QS13 & $(3 \%$, people, Pollution is Low, Smoker is True, Cancer is True) \\
\hline & QS14 & (97\%, people, Pollution is Low, Smoker is True, Cancer is False) \\
\hline & QS15 & (0.1\%, people, Pollution is Low, Smoker is False, Cancer is True) \\
\hline & QS16 & (99.9\%, people, Pollution is Low, Smoker is False, Cancer is False) \\
\hline & QS17 & $(90 \%$, people, Cancer is True, X Ray is Positive) \\
\hline & QS18 & (10\%, people, Cancer is True, XRay is Negative) \\
\hline & QS19 & $(20 \%$, people, Cancer is False, X Ray is Positive) \\
\hline & QS20 & (80\%, people, Cancer is False, XRay is Negative) \\
\hline & QS21 & $(65 \%$, people, Cancer is True, Dyspnoea is Positive) \\
\hline & QS22 & (35\%, people, Cancer is True, Dyspnoea is Negative) \\
\hline & QS23 & (30\%, people, Cancer is False, Dyspnoea is Positive) \\
\hline & QS24 & (70\%, people, Cancer is False, Dyspnoea is Negative) \\
\hline
\end{tabular}

Table 2: Intermediate language expression with crisp quantifiers obtained applying the procedure described in Table 1 to the example of Figure 1

conclusion for the query pair variable-value $P(X=x)$, which corresponds to QS: $Q$ Us are $x$, being $U$ the universe of $X$.

In Algorithm 1 we describe the general procedure for the extraction of the relevant QS (SKB) in the KB for the content determination of the linguistic explanation of the inferred conclusion (query pair variable-value $P(X=x)$ ). In the first place, the $\mathrm{BN}$ is converted into a $\mathrm{KB}$ made up of QS, following the procedure in Table 1. Secondly, the iterative search of the QS is performed. In the first place, all the QS involving value $x$ in their PT are selected (to this aim, the values list is initialised with $x$ ). After that, the corresponding $S T$ are considered as the new values to be iteratively searched for in the $\mathrm{KB}$ and therefore added to the value list. Value $x$ is no longer considered. This is repeated until the values list is empty. At this point, the procedure ends and all the selected QS are returned. These are the QSs which actually correspond to the variables in the ancestors of node $N$ in the BN.

\subsection{Three Scenarios of the Explanation Algorithm Applied to Different Cases of Predictive Reasoning}

Let us consider a computational system that implements the BN of Fig. 1 and a user that interacts with it by means of a natural language. We define three possible scenarios for the interaction between the user and the system that in- clude predictive reasoning with and without evidence. As universe of reference, let us suppose the population of a city, which is labelled as "people".

In the first scenario, the user is just interested in knowing the probability of having cancer, without any other additional information:

User: "What is the chance that I will have cancer?"

System: "Without any other additional information about where do you live or whether or not you are a smoker, you have $1.163 \%$ of chance of having cancer."

User: "Why?"

This question triggers the explainability module. According to Algorithm 1, KB is initialised following the procedure in Table 2 and LIST with "Cancer is True". The $y$ value is "Cancer is True" (query variable and value) and all those statements in $\mathrm{KB}$ that have "Cancer is True" in the PT are extracted, obtaining $S K B=$ $\{Q S 9, Q S 11, Q S 13, Q S 15\}$ after the first iteration of the external loop. In the second and third iterations, the values of $y$ are all the values of variables Pollution and Smoker; therefore, all those statements with these variables in the ST must be added to $\mathrm{SKB}$, obtaining as final result $S K B=\{Q S 1, Q 2 S, Q 3 S, Q S 4, Q S 5, Q S 6, Q S 7, Q S 8, Q S 9$, QS11, QS13, QS15\}. 
Data: $X$ (query variable), $x$ (value of $X$ )

Result: SKB, the selected QS

$\mathrm{KB}=$ Knowledge Base of QS obtained using the

procedure in Table 1;

$\mathrm{N}=|K B|$, the number of QS in $\mathrm{KB}$;

$\mathrm{SKB}=\emptyset$;

LIST $=\{" X=x "\} ;$

while $L I S T<>\emptyset$ do / * still values to look

for in the PTs of the KB * 1

SELECT $y \in$ LIST / * any of the remaining

$\mathrm{PTS} * /$;

for $k=1$ to $N$ do $\quad / *$ looking for $y$ in the

$\mathrm{PT}$ of the $\mathrm{KB} * 1$

if $y$ is a $P T$ of $Q S_{k}$ then

$S K B=S K B \bigcup\left\{Q S_{k}\right\} \quad / * Q S_{k}$ added

to the $\mathrm{KB} * / /$;

LIST $=$ LIST $\bigcup Y$, being $Y$ all the values in

the ST of $Q S_{k} / *$ All the values in

the ST to be considered */;

end

end

LIST $=$ LIST $\backslash y$;

end

Algorithm 1: Algorithm in pseudo-code for the selection of the relevant set of QS for the explanation of a predictive inference without evidence (query variable $X=x$ ).

Once the content determination step is completed (i.e., $\mathrm{SKB}$ ), a template-based linguistic realisation, following the NLG pipeline, is generated for answering the question "Why?". In order to make clear the causal relation supporting the different QS involved in the explanation, we use the linguistic causality marker "because":

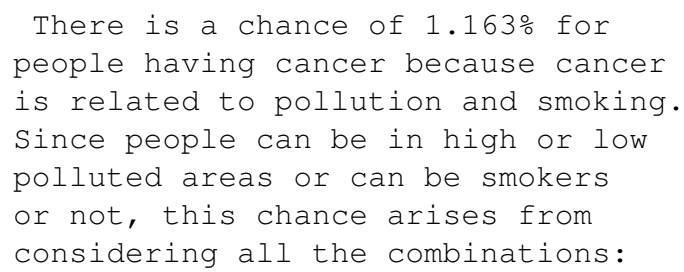

- $0.81 \%$ is the chance for people

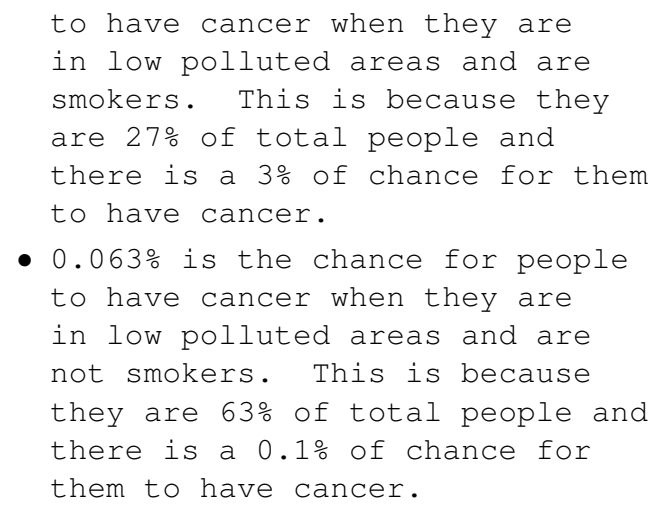

In a second scenario, we address reasoning with evidence when it is in the parent node of the query variable. Thus, without loss of generality, let us consider a node $N$ in a $\mathrm{BN}$, associated to variable $X$ and let $x$ be one of its values. Let $Z$ be the variable in one of the ancestors of $N$ and $z$ one of its values, for which there is some fixed evidence $P(Z=z)=1$. The procedure in this case will obtain the QSs associated to the BN which explain the inferred conclusion $P(X=x \mid Z=z)$, which corresponds to QS: $Q$ zs are $x$.

Thus, the user says to the system that s/he is a smoker (the evidence Smoker is True), obtaining the answer "there is a chance of $3.2 \%$ for smokers to have cancer". This evidence, in addition to be in the parent node of the query variable, it is also in a root node of the network and this simplifies the explanation. Both the QSs of KB about independence and all QSs involving values different of $z$ are also discarded, since the corresponding quantifiers become null. Thus, following the Algorithm 1, the $y$ value is again Cancer is True but the ST is Smoker is True, therefore, we have to select those QSs in the KB satisfying this constraint; obtaining in the first iteration of the external loop $S K B=\{Q S 9, Q S 13\}$. In the next iteration, since the independence QSs are not required, we just have to add the QS corresponding to the other term in the ST of QS9 and QS13; i.e., Pollution is High and Pollution is Low. As a result, $S K B=\{Q S 9, Q S 13, Q S 1, Q S 2\}$.

The generated linguistic summary is the following one:

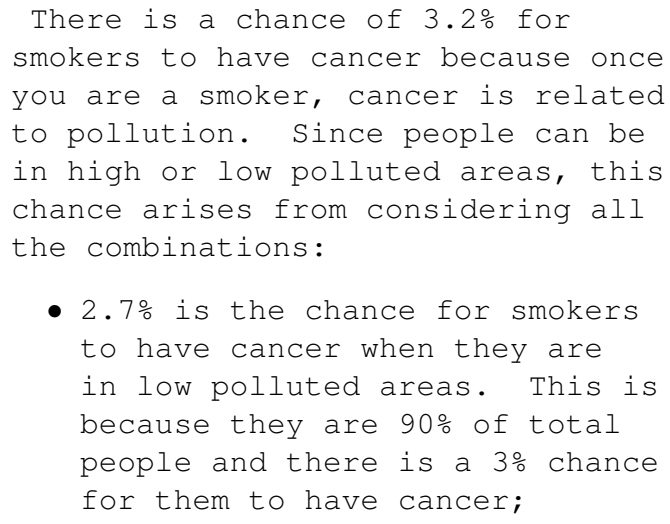




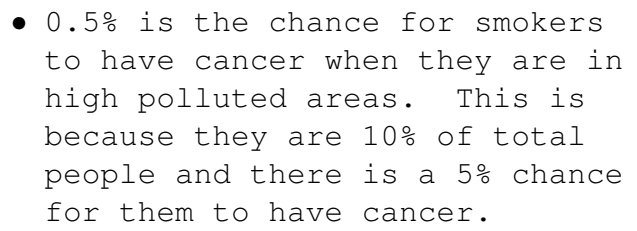

In the third scenario, the only difference with respect to the previous one is that $Z$ is an ancestor which is not a parent of $X$. This case can be solved applying the same procedure. Once again, QSs related to the separation or involving values different of $z$ will be discarded.

Thus, the user asks the system the chances of having dyspnoea given he is a smoker. The system replies "there is a chance of $31.12 \%$ for smokers to have dyspnoea, because dyspnoea is related to cancer". For building the explanation, according to Algorithm 1, the $y$ value in this case is "Dyspnoea is Positive" and the first set of statements are those where $y$ is the PT; i.e., $S K B=\{Q S 21, Q S 23\}$. In the next iteration of the external loop, we add all the QS where the STs of the QS of SKB are PTs taking into the evidence; i.e., "Cancer is True" and "Cancer is False" given "Smoker is True"; i.e., $S K B=\{Q S 14, Q S 13, Q S 10, Q S 09\}$. Finally, $S K B=\{Q S 1, Q S 2\}$ are selected.

The resulting linguistic explanation is as follows:

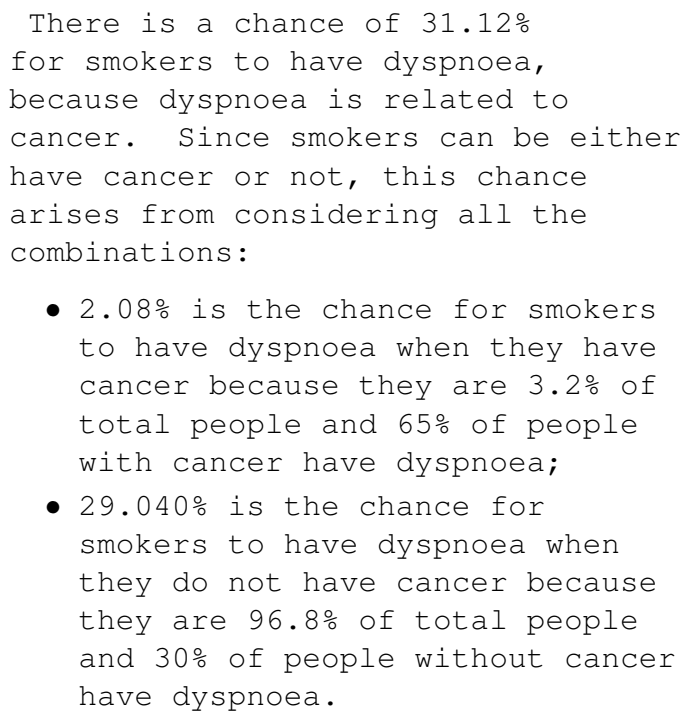

The remaining part of the explanation can be generated in the same way of scenario 2 .

\section{Conclusions}

We have presented a novel method for the explanation of predictive Bayesian Networks (BNs) using a linguistic approach which makes use of the equivalence of BNs and binary Quantified Statements. The method focuses on the content determination stage of Natural Language Generation systems, by generating Quantified Statements (of the form $Q S T$ are $P T$ ) which facilitates the generation of explanations of the reasoning process in BNs in a templatebased NLG system. Particularly, these Quantified Statements make explicit some issues that are implicit in the BNs approximate reasoning mechanism, such are the independence condition for root node variables as well as Dseparation when evidence for input variables is fixed. Since we just focus on the relevant information for the variable in the query, we do not need to explain how all the values of the $\mathrm{BN}$ are updated, which simplifies its interpretability for non-expert users.

For future work, we will address the extension of this framework for dealing with other types of reasoning in BNs, such as inter-causal or diagnostic ones. In the linguistic module, we will address how the use of fuzzy quantifiers can improve the overall quality of the explanations for the users. Finally, we will integrate this framework in a template-based NLG system for automatic generation of explanations in natural language for BN knowledge representation, including an evaluation phase in order to quantify how useful are these explanations for the user and how they can be improved.

We have laid the groundwork for building explanations for BN using a NLG approach, which makes them legible for non-experienced users. We adopted a new viewpoint about the pair node-variable, with the use of binary Quantified Statements, and the reasoning structure, with the chaining between these statements.

\section{Acknowledgement}

This research was funded by the Spanish Ministry for Science, Innovation and Universities (grant TIN2017-84796C2-1-R) and the Galician Ministry of Education, University and Professional Training (grants ED431C 2018/29 and "accreditation 2016-2019, ED431G/08"). All grants were co-funded by the European Regional Development Fund (ERDF/FEDER program). The first author was also funded by the "Consellería de Cultura, Educación e Ordenación Universitaria" under the Postdoctoral Fellowship accreditation ED481B 2016/048-0.

\section{References}

[1] D. V. Aha, T. Darrell, P. Doherty, D. Magazzeni, IJCAI-18 Workshop on Explainable AI (XAI), http://home.earthlink. net/ dwaha/ research/meetings/faim18-xai, last accessed May 15, 2019. (2018).

[2] D. V. Aha, T. Darrell, M. Pazzani, D. Reid, C. Sammut, P. Stone, IJCAI-17 Workshop on Explainable AI (XAI), http://www.intelligentrobots. org/files/IJCAI2017/IJCAI-17_XAI_ WS_Proceedings.pdf, last accessed May 15, 2019. (2017). 
[3] DARPA, Explainable Artificial Intelligence (XAI), https://www.darpa.mil/program/ explainable-artificial-intelligence, last accessed May 15, 2019. (2016).

[4] P. Johnson-Laird, B. G. Bara, Syllogistic inference, Cognition 16 (1984) 1 - 61.

[5] D. Kahneman, Thinking, Fast and Slow, Farrar, Straus and Giroux, 2011.

[6] K. Korb, Bayesian informal logic and fallacy, Informal Logic 23 (2) (2004) 41-70.

[7] K. Korb, R. McConachy, I. Zukerman, A cognitive model of argumentation, in: Proceedings of the Nineteenth Annual Conference of the Cognitive Science Society, 1997, pp. 400-405.

[8] K. Korb, A. Nicholson, Bayesian Artificial Intelligence, Chapman \& Hall/CRC, 2004.

[9] Norsys, Netica application, http://www . norsys.com/netica.html, last accessed May 15, 2019. (2017).

[10] M. Oaksford, N. Chater, Bayesian Rationality. The probabilistic approach to human reasoning, Oxford University Press, Oxford, 2007.

[11] Parliament and Council of the European Union, General data protection regulation (GDPR), http:// data. europa. eu/eli/reg/2016/679/oj, last accessed May 15, 2019. (2016).

[12] M. Pereira-Fariña, A. Bugarín, Approximate syllogism as argumentative expression for knowledge representation and reasoning with generalized Bayes' theorem, in: D. Mohammed, M. Lewinski (Eds.), Argumentation and Reasoned Action: Proceedings of the First European Conference on Argumentation, Studies in Logic and Argumentation. College Publications, 2016, pp. 817-830.

[13] M. Pereira-Fariña, J. C. Vidal, F. Díaz-Hermida, A. Bugarín, A fuzzy syllogistic reasoning schema for generalized quantifiers, Fuzzy Sets and Systems 234 (2014) $79-96$.

[14] E. Reiter, R. Dale, Building Natural Language Generation Systems, Cambridge University Press, 2000

[15] D. G. Schwartz, Dynamic reasoning with qualified syllogisms, Artificial Intelligence 93 (1997) 103-167.

[16] K. van Deemter, E. Krahmer, M. Theune, Squibs and discussions: Real versus template-based natural language generation: A false opposition?, Computational Linguistics 31, 1 (2005) $15-23$.
[17] W. Wiegerinck, M. Nijman, W. ter Burg, E. ter Braak, Y. O, J. Neijt, H. Kappen, Inference and advisory system for medical diagnosis second report STW-NGN55. 3614, http: / /www.snn.ru.nl/ v2/serve. php?doc=eindl. pdf (1999). 